\title{
Graphene Inclusion Induced Ultralow Thermal Conductivity and Improved Figure of Merit in p-type SnSe
}

\author{
Lei Chen ${ }^{\mathrm{a}}$, Weiyao Zhao ${ }^{\mathrm{a}}$, Meng Li $^{\mathrm{a}}$, Guangsai Yang ${ }^{\mathrm{a}}$, Sheik Md Kazi Nazrul \\ Islam $^{\mathrm{a}}$, David R.G. Mitchell ${ }^{\mathrm{b}}$, Zhenxiang Cheng ${ }^{\mathrm{a}}$, Xiaolin Wang ${ }^{\mathrm{a}^{*}}$
}

a Institute for Superconducting and Electronic Materials, Australian Institute for Innovative Materials, University of Wollongong, North Wollongong, 2500, Australia

b Electron Microscopy Centre, Australian Institute for Innovative Materials, University of Wollongong, North Wollongong, NSW 2500, Australia

\section{Abstract}

The concept of composite material has been increasingly applied for the significant improvement on the thermoelectric performance because of the predictable effective medium properties and the unique interfacial correlated thermal and electrical transport mechanism. Herein, we report that the graphene inclusion can lead to significant reduction in thermal conductivity and improve the overall thermoelectric figure-of-merit in SnSe. We demonstrate a systematic investigate on the microstructures, electrical and thermoelectric properties of the $\mathrm{SnSe} /$ graphene composite. HRTEM reveals uniform distribution of graphene nanosheets in the SnSe matrix, forming sharp interface with refined SnSe grain sizes and defects nearby the interfaces. Thermal conductivity decreases with graphene addition and can significantly reduce to as low as $\sim 0.18 \mathrm{Wm}^{-1} \mathrm{~K}^{-}$,

\footnotetext{
*xiaolin@uow.edu.au
} 
resulting in an enhanced figure of merit $(Z T)$ of $\mathrm{SnSe} /$ graphene composite by at least $50 \%$ compared with pristine SnSe. The significant reduction on thermal conductivity is attributed to the phonon scattering by the densely distributed phase interfaces as well as defects and grain boundaries. The carbon element is also believed to potentially reducing long-range tin diffusion by acting as a confinement barrier to restrict heat and ion diffusion. Our work proves that graphene secondary phase could enhance the $Z T$ of SnSe matrix, which might pave ways for achieving high-performance thermoelectric in carbon-induced composite materials.

\section{Introduction:}

Thermoelectric technology, which can directly convert waste heat into electrical energy, has been actively considered for a variety of energy harvesting and thermal management applications ${ }^{1-3}$. The efficiency of thermoelectric modules is often evaluated by a dimensionless figure-of-merit $Z T=S^{2} \sigma T / \kappa$, where $S$ is Seebeck coefficient, $\sigma$ is electrical conductivity, $T$ is the absolute temperature, and $\kappa=\kappa_{e}+\kappa_{l}$ is the thermal conductivity, constituted by electronic $\kappa_{e}$ and lattice $\kappa_{l}$ contributions, respectively ${ }^{4}$. To screen thermoelectric materials with high $Z T$, both high power factor $\left(S^{2} \sigma\right)$ and/or low $\kappa$ are needed. So far, significant breakthroughs have been made in improving $S^{2} \sigma$ via resonant state doping ${ }^{5,6}$, band convergence ${ }^{7,8}$, quantum confinement ${ }^{9,10}$, and minority carrier blocking ${ }^{11,12}$. The thermal conductivity $\kappa$ can also be significantly reduced via strengthening phonon scattering by hierarchical architecturing ${ }^{13-15}$, nanostructuring ${ }^{16,17}$, and introducing nanoprecipitates and defects to the matrix ${ }^{18}$, ${ }^{19}$. However, some competing correlations (e.g. $S, \sigma$ and $\kappa$ are inversely interacted through carrier concentration $(n)$ ) preventing these parameters from being unlimited optimized ${ }^{20}$. 
IV-VI binary compounds have been considered as ideal mid- and hightemperature thermoelectric materials due to their appropriate semiconducting gap and/or phase transition phenomenon. Historically, symmetric rock-salt structured $(F m \overline{3} m)$ IV-VI compounds such as $\mathrm{PbTe}^{21-23}, \mathrm{PbSe}^{24-26}, \mathrm{GeTe}^{27,28}, \mathrm{SnTe}^{29-32}$, and their derivatives or alloys have been substantially studied because they usually have large band degenerancy $\left(N_{v}\right)$ and effective mass $\left(m^{*}\right)$, securing high quality factor $B$ ( $B$ is the material quality factor that related only with the inherent material properties) which determines high ZT. However, environmental and/or economic issues prohibit them from mass-market application. During last decade, studies of another series of IV-VI compounds having highly anisotropic layered orthorhombic structure (Pnma), such as $\mathrm{SnSe}^{17,33}, \mathrm{SnS}^{34-36}$ and $\mathrm{GeSe}^{37,38}$, have flourishingly emerged. Recently, record-high $Z T$ has been reported in single crystal SnSe, namely $\sim 2.6$ at $923 \mathrm{~K}$ along the b-axis of $p$-type vacancies doped $\mathrm{SnSe}^{33}$. An impressive energy conversion efficiency can be predicted over $16 \%$ if applying $500 \mathrm{~K}$ temperature difference to a thermoelectric module consisting of as-reported materials ${ }^{39}$, which stimulates renewed interests in the SnSe-based thermoelectric materials. Compared with single crystal SnSe suffering from reported poor mechanical robustness and prospective high production, polycrystalline SnSe bulks are more feasible for practical application. Cuttingedge studies have considered the microstructural factors ${ }^{28,40-42}$ (e.g. preferential orientation and texture) and influence on the electron and phonon tensors denoting thermoelectric performance ${ }^{43-45}$, while mainly stressed in optimizing the aforementioned $n$ and $\kappa$ to enhance $Z T$. Documented effective strategies towards enhancing $Z T$ of polycrystalline SnSe include resonant doping ${ }^{4,46,47}$, band structure engineering ${ }^{12,48}$, and nanostructuring ${ }^{17}$.

More studies for enhancing $Z T$ also focus on extrinsically embedding alien phase in thermoelectric matrix to form composite materials. The thermoelectric properties of acquired composite can be roughly estimated according to the 
effective medium theory ${ }^{49,50}$ (despite the interfacial influence is underestimated), and can show some anomalous behaviour depending on the intrinsic properties of the embedded phase ${ }^{51,52}$. The recent independent researches show significant enhancement of $Z T$ of $\mathrm{Cu}_{2} \mathrm{Se}$ to over 2.4 by incorporating graphitic phase, such as carbon nanotube and graphene, to $\mathrm{Cu}_{2} \mathrm{Se}$ matrix ${ }^{53-56}$. The significant enhancement of $Z T$ is attributed to the great suppress of phonon propagation when heat vibration passes through the phase interfaces. It is also found that the graphitic phase can act as "ionic filter" which can enhance the stability of thermoelectric materials. ${ }^{57}$ Later on, enhanced $Z T$ have also been achieved in other thermoelectric materials, such as $\mathrm{Bi}_{2} \mathrm{Te}_{3}{ }^{58}, \mathrm{Cu}_{2} \mathrm{~S}^{57}$, etc. These works jointly confirm that graphitic phase can slightly affect the $S^{2} \sigma$, while drastically reduce the $\kappa$, leading to enhanced $Z T$ of composite materials. Analogously, it is reasonable to predict an enhanced $Z T$ in $\mathrm{SnSe} / \mathrm{C}$ composite materials considering the scientificity and universality of this strategy.

In this work, we successfully synthesized graphene incorporated SnSe thermoelectric materials via spark plasma sintering. The as-acquired $\mathrm{SnSe} /$ graphene composites show enhanced $Z T$ at arbitrary temperature compared with pristine SnSe, reaching a peak value of 1.06 at $823 \mathrm{~K}$. The enhanced $Z T$ is primarily governed by the ultralow thermal conductivity down to $\sim 0.18 \mathrm{Wm}^{-1} \mathrm{~K}^{-}$ 1, which is attributed to the phonon scattering by the densely distributed phase interfaces as well as defects and grain boundaries. The carbon element is also believed to potentially reducing long-range tin diffusion by acting as a confinement barrier to restrict heat and ion diffusion ${ }^{53}$. Our work proves that graphene secondary phase could enhance the $Z T$ of SnSe matrix, which might pave ways for achieving high-performance thermoelectric in carbon-induced composite materials.

\section{Synthesis and Measurements}


The different graphene-concentration samples were prepared by directly mixing SnSe and different weight fraction of graphene in an agate mortar with ethanol, then grinding the mixture into fine powders. The SnSe and graphene were mixed in the weight ratios of $1: x(x=0.1 \%, 0.3 \%, 0.5 \%$, and $1 \%)$, respectively. The powders were then loaded in a graphite die in diameter of 20 $\mathrm{mm}$ and densified by spark plasma sintering (SPS) at $773 \mathrm{~K}$ for 5 min under a uniaxial pressure of $45 \mathrm{MPa}$. The obtained polycrystalline bulks were cut into round disks and rectangular bars and polished for thermal and electronic measurements along the direction of the SPS pressure, respectively.

The crystal structure was investigated by X-ray diffraction (XRD) performed on a MAC Science system using $\mathrm{Cu} \mathrm{K}_{\alpha}$ radiation within the $2 \theta$ range from $20^{\circ}$ to $60^{\circ}$ at room temperature. Scanning electron microscopy (SEM) and scanning transmission electron microscopy (STEM) were then used to further deduce the morphology of graphene in the SnSe bulks.

The Seebeck coefficient and electrical conductivity were measured simultaneously using a commercial RZ2001i system from room temperature to $830 \mathrm{~K}$ under vacuum. Thermal conductivity was determined in the 300-830 K temperature range by combining the thermal diffusivity $D$, specific heat $C_{p}$, and density $d$ according to the relation $\kappa=D C_{p} d$, where $\kappa=\kappa_{L}+\kappa_{e}$ is the total thermal conductivity, and $\kappa_{L}, \kappa_{e}$ are the lattice and electron contribution respectively. The thermal diffusivity $(D)$ was measured by the laser flash technique (LINSEIS LFA 1000) under vacuum conditions. The specific heat $\left(C_{p}\right)$ was determined by differential scanning calorimetry on a DSC-204F1 Phoenix under argon atmosphere with a flow rate of $50 \mathrm{ml} / \mathrm{min}$. The sample density $(d)$ was calculated from the measured mass and dimensions and was considered temperature-independent. All the electrical conductivity and thermal diffusivity measurements on the as-prepared polycrystalline bulks were repeated several times to confirm their reproducibility. 
The room temperature Hall carrier concentration $\left(n_{\mathrm{H}}\right)$ was measured using a Quantum Design Physical Properties Measurement System (PPMS). The fourcontact Hall-bar geometry was used for the measurements. The carrier concentration $(n)$ and carrier mobility $(\mu)$ were calculated from the formula $n=1 /\left(\mathrm{eR}_{\mathrm{H}}\right)$ and $\mu=\sigma \mathrm{R}_{\mathrm{H}}$, where e and $\sigma$ are the electron charge and the electrical conductivity, respectively, and $\mathrm{R}_{\mathrm{H}}$ is the Hall coefficient.

\section{Results and Discussion}

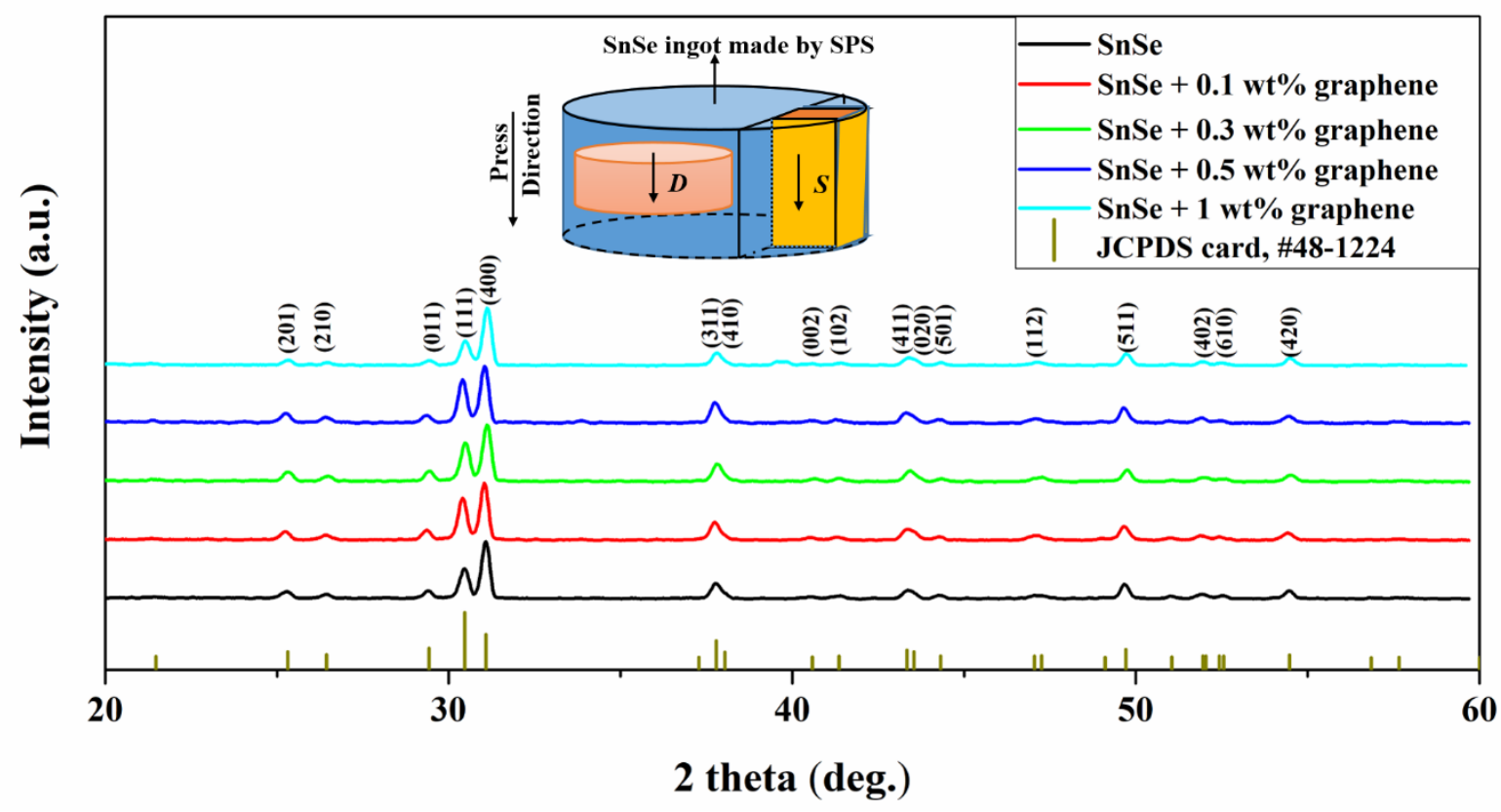

Fig.1 XRD patterns of SnSe samples with different graphene concentrations. Inset is the synthesis procedure, schematic diagram of inner structure and sample cutting for thermal diffusivity $(D)$, and Seebeck $(S)$ and electrical conductivity measurements, respectively.

Fig. 1 shows the room-temperature XRD patterns of the SnSe samples with various graphene concentrations. All diffraction peaks can be well indexed to the orthorhombic SnSe phase with the space group Pnma (JCPDS card, 48-1224), indicating that graphene does not introduce any additional impurity phases and forms composite with SnSe. Fig. 2(a) is the SEM backscattering characterization on SnSe sample with 0.5 wt.\% graphene, and the inset picture of Fig. 2(a) shows the SEM image on the fractography of the same sample. The fractured surface 
shows a highly compacted structure. Fig. 2(b), (c), (d) are the elemental mapping results for the area of the sample in Fig. 2(a), where the elements Sn and Se are distributed uniformly, and the carbon is also mixed well with $\mathrm{SnSe}$, with preferred aggregation near the grain boundary. No large carbon clusters were observed in our samples. All these results indicate that the graphene is incorporated into the SnSe matrix, forming a carbon-phase reinforced nanocomposite structure.

Fig. 3(a) is the transmission electron microscope (TEM) image of the $\mathrm{SnSe} /$ graphene interface in the $0.5 \mathrm{wt} . \%$ graphene incorporated SnSe sample, from which the SnSe/graphene nano-sized boundaries can be observed. Fig. 3(b) is the transmission electron microscope image at high resolution (HRTEM) of the circled area in Fig. 3(a) in the bright field (BF) mode, in which the interplanar lattice spacings of $5.75 \AA$ and $3.03 \AA$ along the [200] and [011] crystallographic directions of SnSe can be found, respectively. The bright area next to SnSe in Fig. 3(b), which shows layered structure, is determined to be graphene. The above results indicate that the interface of $0.5 \mathrm{wt} . \%$ graphene incorporated SnSe sample contains only one phase of $\mathrm{SnSe}$ and that the interface is formed by $\mathrm{SnSe}$ and graphene, which means that the carbon does not go into the SnSe lattice, but mixes with the matrix and contributes to the formation of many boundaries for phonon scattering. These results are in good agreement with the XRD analysis. It is clear that the graphene is indeed dispersed in the SnSe matrix to form the heterogeneous interfaces. It is suggested that the incorporated graphene could suppress atom diffusion during sintering and restrict the grain size. It should also be noted that some cracks are found to be distributed along the grain boundaries, which should be attributed to the great difference between the thermal expansion parameters of carbon and SnSe. 


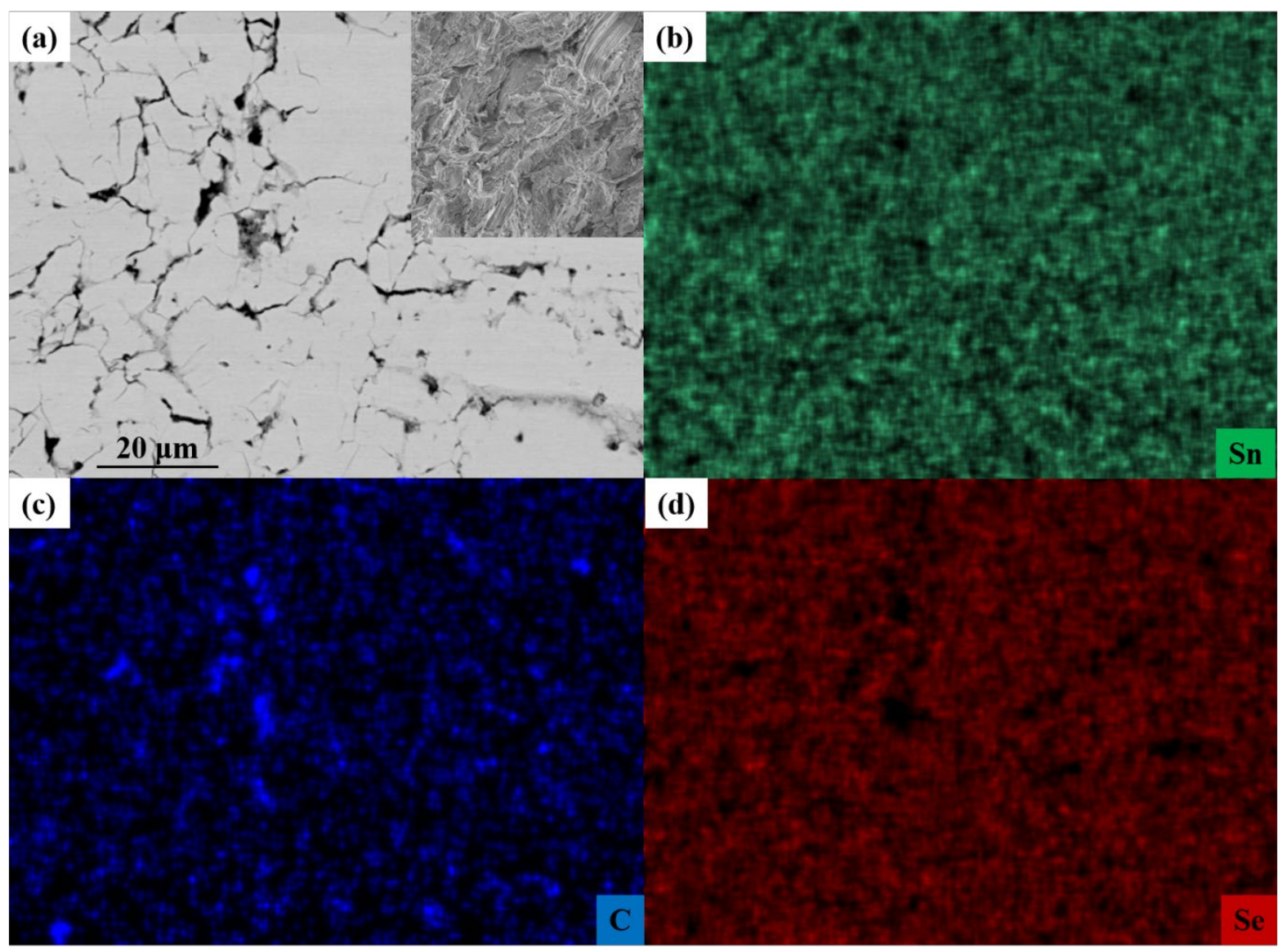

Fig. 2(a) SEM backscattering characterization on SnSe sample with $0.5 \mathrm{wt} \%$ graphene, inset is the SEM image of fracture section on the same sample. (b), (c), (d) SEM elemental mapping results of the area in (a).

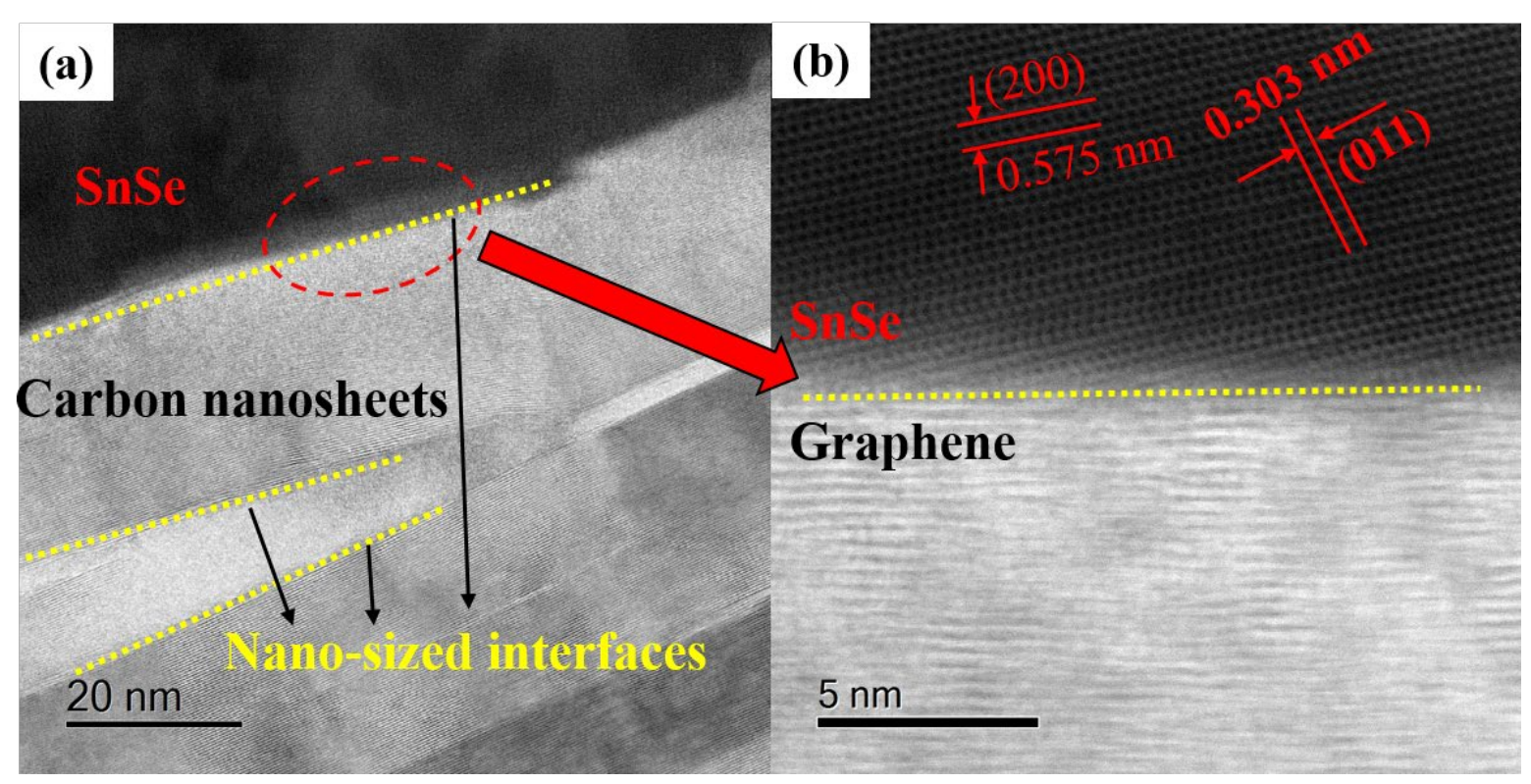


Figure 3 (a) TEM image of nano-sized SnSe/graphene interfaces in $0.5 \mathrm{wt} \%$ graphene incorpotated SnSe sample. (b) BF image of the circled area in (a)

According to the representative areas shown in Fig. 3, there are many nanoscale boundaries between SnSe and graphene. Such specific nanostructures are proposed to be formed due to the combined effects of the graphene network and the nature of the processing. In fact, complex nanostructures are often observed in other materials synthesized by SPS or other fabrication methods such as mechanical alloying (MA) ${ }^{55,59}$. Nevertheless, such nanostructures can exhibit certain advantages, for instance, increased phonon scattering and electron filtering at grain boundaries, better mechanical properties, and improved isotropy ${ }^{53}$. These are proved by our extremely low thermal conductivity and relatively high $Z T$.
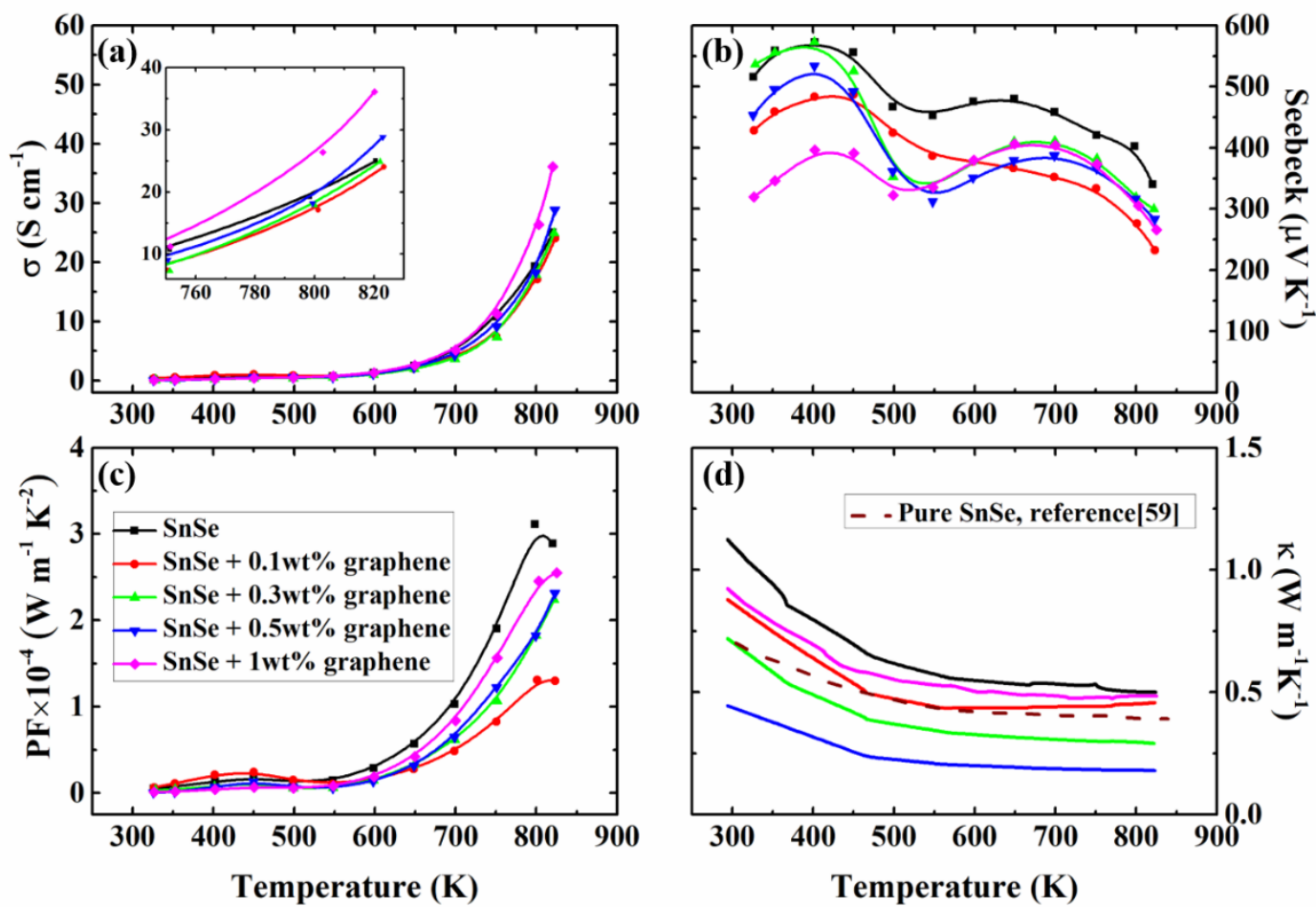
Figure 4 Temperature dependent (a) electrical conductivity, inset is details at high temperature, (b) Seebeck coefficient, (c) power factor, and (d) thermal conductivity for SnSe samples with different graphene content, respectively. The dashed line in $(d)$ is thermal conductivity for pure polycrystalline SnSe plotted from reference work ${ }^{60}$.

The temperature dependences of the electrical conductivity $(\sigma)$, Seebeck coefficient $(S)$, power factor $(\mathrm{PF})$, and thermal conductivity $(\kappa)$ are shown in Fig. 4(a), (b), (c), and (d), respectively, and the inset of Fig. 4(a) shows details of electric conductivity at high temperature. On one hand, the electrical conductivity $\sigma$ shows an overall increase with increasing temperature for all the samples, indicative of semiconducting behaviour. On the other hand, $\sigma$ decreases slightly in the $0.1 \mathrm{wt} . \%$ and $0.3 \mathrm{wt} \%$ graphene doped SnSe samples, then clearly increases for the other two high-level graphene-doped samples, compared with undoped SnSe. The reduction of electrical conductivity in low-doped SnSe is regard as a result of defects introduced by the dopant, while the enhancement of $\sigma$ in the highlevel doped samples is contributed mostly by the improved carrier mobility (Fig. $5(\mathrm{a}))$.

The values of $S$ are positive over the whole temperature range, suggesting that holes are the majority charge carriers (Fig. 4(b)), so the samples are $p$-type. For pure $\mathrm{SnSe}$, the Seebeck coefficient is $516 \mu \mathrm{V} \mathrm{K}^{-1}$ at $326 \mathrm{~K}$, and it increases to $572 \mu \mathrm{V} \mathrm{K}^{-1}$ at $402 \mathrm{~K}$, at which point it starts to decrease rapidly with increasing temperature up to $573 \mathrm{~K}$. It then increases slightly up to $673 \mathrm{~K}$ and finally decreases. Our pure SnSe sample has a similar maximum Seebeck value and trend in its temperature dependence to previously reported $p$-type $\mathrm{SnSe}^{60}$. The Seebeck coefficient decreases gradually with increasing graphene concentration. It drops from $516 \mu \mathrm{V} \mathrm{K}^{-1}$ in pure SnSe to $319 \mu \mathrm{V} \mathrm{K}^{-1}$ in $1 \mathrm{wt} . \%$ graphene added SnSe at $325 \mathrm{~K}$. All the graphene-added samples show a similar trend of the Seebeck coefficient with temperature. 
Fig. 4(d) shows the temperature dependence of the total thermal conductivity $\kappa$ with graphene addition. The $\kappa$ values of all samples are lower than that of the pure SnSe. The lowest $\kappa$ is achieved in the $0.5 \mathrm{wt} . \%$ graphene added SnSe sample, with the values of $0.44 \mathrm{~W} \cdot \mathrm{m}^{-1} \mathrm{~K}^{-1}$ at $294 \mathrm{~K}$ and $0.18 \mathrm{~W} \cdot \mathrm{m}^{-1} \mathrm{~K}^{-1}$ at $823 \mathrm{~K}$, respectively. The latter is much lower than that in single-crystal SnSe, which has a $\kappa$ of $0.23 \pm 0.03 \mathrm{Wm}^{-1} \mathrm{~K}^{-1}$ at $973 \mathrm{~K}^{33}$. Compared to state-of-the-art thermoelectric compounds, this thermal conductivity value is exceedingly low ${ }^{61}$. Also, this remarkably low value is lower than those obtained even by nanostructuring and all-scale hierarchical architectures of PbTe-based thermoelectric materials ${ }^{13}$. The presence of graphene in SnSe leads to a decrease in the total thermal conductivity. Obviously, this is because the phonon scattering is enhanced due to the formation of multiple defects as well as interfaces and boundaries between the SnSe matrix and graphene at the nanoscale level. Previous studies have proved that, the generalized phonon density of states (GDOS) of graphene and SnSe are quite different from each other, e.g., for SnSe, the phonons are mainly populated below $\sim 25 \mathrm{meV}^{62}$; for graphene, the phonons populate to above $200 \mathrm{meV}^{63}$. In the present $\mathrm{SnSe} /$ graphene composite, the nanoscale interfaces provide good combination between two lattices. However, the lattice vibration can only pass the interface with low efficient because of the widely divergent lattice dynamics, thus the heat cannot spread efficiently in the composite, resulting in low thermal conductivity. Such defects, interfaces, and boundaries induced by graphene doping were also observed in $\mathrm{Cu}_{2} \mathrm{Se} \mathrm{systems}^{53}$. 


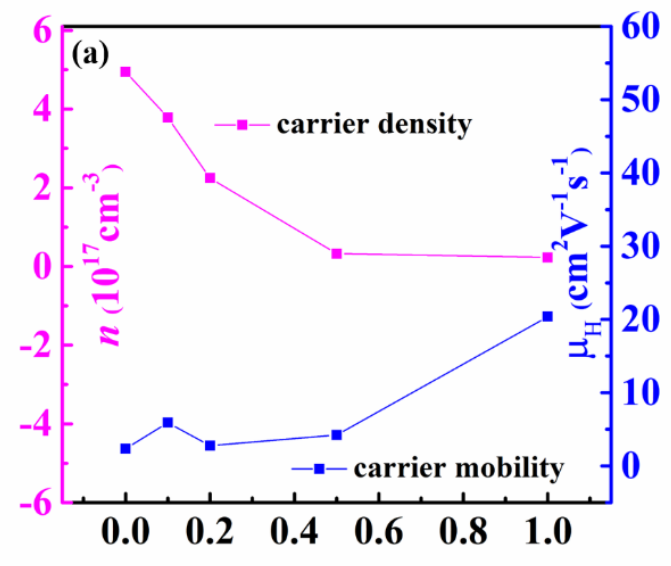

Graphene Content (wt.\%)

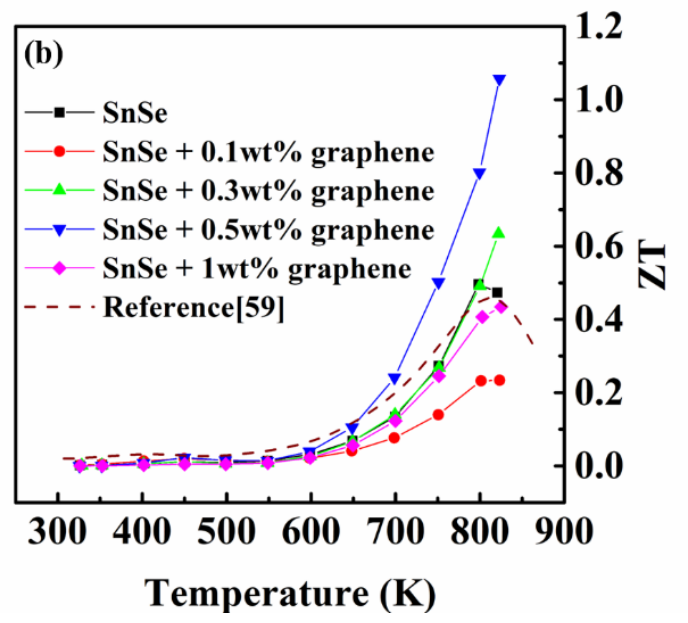

Temperature (K)

Figure 5 (a) Carrier density and mobility for all of the SnSe/graphene composite samples at room temperature. (b) Temperature dependent $Z T$ values for SnSe samples with different graphene contents, the dashed line is previously reported pure polycrystalline $\mathrm{SnSe}^{60}$.

In order to understand the behaviour of electronic transport, room temperature Hall-effect measurements have been performed on all of the $\mathrm{SnSn/graphene} \mathrm{composite} \mathrm{samples} \mathrm{to} \mathrm{measure} \mathrm{the} \mathrm{carrier} \mathrm{density.} \mathrm{The} \mathrm{carrier}$ concentration $(n)$ decreased from $4.9 \times 10^{17} \mathrm{~cm}^{-3}$ to $2.3 \times 10^{16} \mathrm{~cm}^{-3}$ with the increasing graphene content. The Pisarenko line was also plotted based on the calculation of the single parabolic band (SPB) $\operatorname{model}^{64,65}$ using the equation $\mathrm{S}=$ $\frac{8 \pi^{2} k_{B}{ }^{2}}{3 e h^{2}} m^{*} T\left(\frac{\pi}{3 n}\right)^{\frac{2}{3}}$, where $k_{\mathrm{B}}$ is Boltzmann constant, $h$ is the Planck constant, and $\mathrm{m}^{*}$ is the band effective mass. By combining the experimental results and the Pisarenko line, we finally got the effective mass of $\mathrm{m}^{*}=0.85 \mathrm{~m}_{0}$, and the result is shown in S1. Fig. 5(b) presents the temperature dependence of the $Z T$ values for all the samples in this work as well as that reported in previous studies on polycrystalline $p$-type $\mathrm{SnSe}^{60}$ (shown as dashed line in Fig. 5 (b)). The ZT of our pure SnSe sample was very close to that in the previous work, and a highest $Z T$ of 1.06 at $823 \mathrm{~K}$ was achieved in the $0.5 \mathrm{wt} \%$ graphene added $\mathrm{SnSe}$, with an enhancement ratio of $125 \%$, indicating that graphene addition is a promising 
environmentally-friendly, economical, and efficient method to improve the thermoelectric performance of polycrystalline SnSe.

Furthermore, the nano-carbon refined thermoelectric materials have proved their capability in great performance enhancement, such as carbon nano tube added $\mathrm{CoSb}_{3}{ }^{66}$, carbon nano tube or graphene added $\mathrm{Cu}_{2} \mathrm{Se}^{55,56}$, and the present graphene doped SnSe. The improvement in thermoelectric behaviour are mainly on two aspects: 1) during material synthesis/moulding, the nano-size carbon restricts the grain size to form more boundaries, 2) carbon materials possess very steep acoustic phonons and extra high optical phonons $(150-250$ $\mathrm{meV}$ ), which mismatches the phonon modes of current thermoelectric materials, and thus limit the heat spreading via lattice dynamics. In the present work, we observed both of the effects: the fine-tuned nano structures in the composite, as well as the decreased thermal conductivity, which reasonably increases the $Z T$ value by $\sim 125 \%$. On the other hand, carbon is one of the most common and cheap element in the world, which benefits the commercialization of nano-carbon refined thermoelectric materials/devices. Besides, the complicate but interesting interface lattice dynamics may also inspire more fundamental studies like inelastic X-ray/neutron spectroscopy investigations. Based on the great $Z T$ enhancement in graphene added SnSe composite, we are confident to expect the extensive use of nano-carbon materials in thermoelectric industry.

\section{Conclusion}

In summary, $p$-type polycrystalline $\mathrm{SnSe}$ incorporated with graphene was prepared by spark plasma sintering. The graphene had a significant effect on the electrical as well as thermal performances. 1) The thermal conductivity decreased greatly with graphene incorporation. An ultra-low thermal conductivity of 0.18 $\mathrm{W} \cdot \mathrm{m}^{-1} \mathrm{~K}^{2}$, which is lower than that in single crystal $\mathrm{SnSe}$, was realised at $823 \mathrm{~K}$ in the SnSe sample with 0.5 wt.\% graphene added in. 2) The Seebeck coefficients 
were also reduced by graphene, which corresponded with the enhanced electrical conductivity. 3) The electrical conductivity was obviously enhanced with graphene doping over the whole temperature range. As a result of the competition among the above parameters, the highest $Z T$ of 1.06 was obtained at $823 \mathrm{~K}$ in 0.5 wt.\% graphene-added SnSe. Compared with intrinsic polycrystalline SnSe, the performance is enhanced by 125 percent excitingly. This graphene added SnSe method would also be generalized to other nano-carbon materials, and other thermoelectric materials, to inspire fundamental dynamics researches, as well as to better sever the energy industry.

\section{Acknowledgements}

This work was partially supported by the Australian Research Council (ARC) through ARC Professorial Future Fellowship project (FT 130100778, XLW) and a Linkage Infrastructure Equipment and Facilities (LIEF) Grant (LE120100069, XLW). Dr. Mitchell Nancarrow of the UOW EMC is thanked for assistance with sample preparation and SEM analysis. This research used equipment (FIB and STEM) funded by the ARC LIEF grants: LE160100063 and LE120100104 respectively, located at the UOW Electron Microscopy Centre. This research was undertaken with the assistance of resources and services from the National Computational Infrastructure (NCI), which is supported by the Australian Government. 


\section{References}

1. $\quad$ H. Goldsmid, Thermoelectric refrigeration, Springer, 2013.

2. B. Poudel, Q. Hao, Y. Ma, Y. Lan, A. Minnich, B. Yu, X. Yan, D. Wang, A. Muto and D. Vashaee, Science, 2008, 320, 634-638.

3. D. M. Rowe, Thermoelectrics and its Energy Harvesting, 2-Volume Set, CRC press, 2018.

4. C.-L. Chen, H. Wang, Y.-Y. Chen, T. Day and G. J. Snyder, Journal of Materials Chemistry A, 2014, 2, 11171-11176.

5. J. P. Heremans, V. Jovovic, E. S. Toberer, A. Saramat, K. Kurosaki, A. Charoenphakdee, S. Yamanaka and G. J. Snyder, Science, 2008, 321, 554-557.

6. M. Hong, Z. G. Chen, L. Yang, Y. C. Zou, M. S. Dargusch, H. Wang and J. Zou, Advanced materials, 2018, 30, 1705942.

7. Y. Pei, X. Shi, A. LaLonde, H. Wang, L. Chen and G. J. Snyder, Nature, 2011, 473, 66-69.

8. X. Liu, T. Zhu, H. Wang, L. Hu, H. Xie, G. Jiang, G. J. Snyder and X. Zhao, Advanced Energy Materials, 2013, 3, 1238-1244.

9. Y. Tian, M. R. Sakr, J. M. Kinder, D. Liang, M. J. MacDonald, R. L. Qiu, H.-J. Gao and X. P. Gao, Nano letters, 2012, 12, 6492-6497.

10. T. Harman, P. Taylor, M. Walsh and B. LaForge, science, 2002, 297, 2229-2232.

11. H. Yang, J.-H. Bahk, T. Day, A. M. Mohammed, G. J. Snyder, A. Shakouri and Y. Wu, Nano letters, 2015, 15, 1349-1355.

12. J.-H. Bahk and A. Shakouri, Physical Review B, 2016, 93, 165209.

13. K. Biswas, J. He, I. D. Blum, C.-I. Wu, T. P. Hogan, D. N. Seidman, V. P. Dravid and M. G. Kanatzidis, Nature, 2012, 489, 414-418.

14. G. Han, Z.-G. Chen, L. Yang, M. Hong, J. Drennan and J. Zou, ACS applied materials \& interfaces, 2014, 7, 989-995.

15. S. Bhattacharya, A. Bohra, R. Basu, R. Bhatt, S. Ahmad, K. Meshram, A. Debnath, A. Singh, S. K. Sarkar and M. Navneethan, Journal of Materials Chemistry A, 2014, 2, 17122-17129.

16. M. Hong, T. C. Chasapis, Z.-G. Chen, L. Yang, M. G. Kanatzidis, G. J. Snyder and J. Zou, ACS nano, 2016, 10, 4719-4727.

17. X. Shi, A. Wu, W. Liu, R. Moshwan, Y. Wang, Z.-G. Chen and J. Zou, ACS nano, 2018, 12, 11417-11425.

18. Y. Pei, J. Lensch-Falk, E. S. Toberer, D. L. Medlin and G. J. Snyder, Advanced Functional Materials, 2011, 21, 241-249.

19. M. H. Lee, J.-S. Rhyee, M. Vaseem, Y.-B. Hahn, S.-D. Park, H. Jin Kim, S.-J. Kim, H. J. Lee and C. Kim, Applied Physics Letters, 2013, 102, 223901.

20. M. H. Elsheikh, D. A. Shnawah, M. F. M. Sabri, S. B. M. Said, M. H. Hassan, M. B. A. Bashir and M. Mohamad, Renewable and sustainable energy reviews, 2014, 30, 337-355.

21. Y. Pei, A. LaLonde, S. Iwanaga and G. J. Snyder, Energy \& Environmental Science, 2011, 4, 2085-2089.

22. A. D. LaLonde, Y. Pei and G. J. Snyder, Energy \& Environmental Science, 2011, 4, 2090-2096.

23. H. Wang, Y. Pei, A. D. LaLonde and G. J. Snyder, Advanced Materials, 2011, 23, 1366-1370.

24. H. Wang, Y. Pei, A. D. LaLonde and G. J. Snyder, Proceedings of the National Academy of Sciences, 2012, 109, 9705-9709.

25. D. Parker and D. J. Singh, Physical Review B, 2010, 82, 035204.

26. Q. Zhang, F. Cao, W. Liu, K. Lukas, B. Yu, S. Chen, C. Opeil, D. Broido, G. Chen and Z. Ren, Journal of the American chemical society, 2012, 134, 10031-10038.

27. D. Wu, L.-D. Zhao, S. Hao, Q. Jiang, F. Zheng, J. W. Doak, H. Wu, H. Chi, Y. Gelbstein and C. Uher, Journal of the American Chemical Society, 2014, 136, 11412-11419.

28. Y. Fu, J. Xu, G.-Q. Liu, J. Yang, X. Tan, Z. Liu, H. Qin, H. Shao, H. Jiang and B. Liang, Journal of Materials Chemistry C, 2016, 4, 1201-1207.

29. R. Moshwan, L. Yang, J. Zou and Z. G. Chen, Advanced Functional Materials, 2017, 27, 1703278. 
30. G. Tan, L.-D. Zhao, F. Shi, J. W. Doak, S.-H. Lo, H. Sun, C. Wolverton, V. P. Dravid, C. Uher and M. G. Kanatzidis, Journal of the American Chemical Society, 2014, 136, 7006-7017.

31. M. Zhou, Z. M. Gibbs, H. Wang, Y. Han, C. Xin, L. Li and G. J. Snyder, Physical Chemistry Chemical Physics, 2014, 16, 20741-20748.

32. G. Tan, F. Shi, S. Hao, H. Chi, L.-D. Zhao, C. Uher, C. Wolverton, V. P. Dravid and M. G. Kanatzidis, Journal of the American Chemical Society, 2015, 137, 5100-5112.

33. L.-D. Zhao, S.-H. Lo, Y. Zhang, H. Sun, G. Tan, C. Uher, C. Wolverton, V. P. Dravid and M. G. Kanatzidis, Nature, 2014, 508, 373-377.

34. W. He, D. Wang, H. Wu, Y. Xiao, Y. Zhang, D. He, Y. Feng, Y.-J. Hao, J.-F. Dong and R. Chetty, Science, 2019, 365, 1418-1424.

35. Z. Wang, D. Wang, Y. Qiu, J. He and L.-D. Zhao, Journal of Alloys and Compounds, 2019, 789, 485-492.

36. S. Roychowdhury, R. K. Biswas, M. Dutta, S. K. Pati and K. Biswas, ACS Energy Letters, 2019, 4, 1658-1662.

37. J. Cui, J. He and Y. Chen, ACS Applied Energy Materials, 2019, 2, 3703-3707.

38. B. U. Haq, S. AlFaify, A. Laref, R. Ahmed and M. Taib, Ceramics International, 2019, 45, 15122-15127.

39. L.-D. Zhao, G. Tan, S. Hao, J. He, Y. Pei, H. Chi, H. Wang, S. Gong, H. Xu and V. P. Dravid, Science, 2016, 351, 141-144.

40. D. Feng, Z.-H. Ge, D. Wu, Y.-X. Chen, T. Wu, J. Li and J. He, Physical Chemistry Chemical Physics, 2016, 18, 31821-31827.

41. Y. Li, F. Li, J. Dong, Z. Ge, F. Kang, J. He, H. Du, B. Li and J.-F. Li, Journal of Materials Chemistry C, 2016, 4, 2047-2055.

42. S. R. Popuri, M. Pollet, R. Decourt, F. D. Morrison, N. S. Bennett and J.-W. G. Bos, Journal of Materials Chemistry C, 2016, 4, 1685-1691.

43. C. W. Li, J. Hong, A. F. May, D. Bansal, S. Chi, T. Hong, G. Ehlers and O. Delaire, Nature Physics, 2015, 11, 1063-1069.

44. R. Guo, X. Wang, Y. Kuang and B. Huang, Physical Review B, 2015, 92, 115202.

45. S. Yang, Y. Liu, M. Wu, L.-D. Zhao, Z. Lin, H.-c. Cheng, Y. Wang, C. Jiang, S.-H. Wei and L. Huang, Nano Research, 2018, 11, 554-564.

46. Y. X. Chen, Z. H. Ge, M. Yin, D. Feng, X. Q. Huang, W. Zhao and J. He, Advanced Functional Materials, 2016, 26, 6836-6845.

47. S. Chen, K. Cai and W. Zhao, Physica B: Condensed Matter, 2012, 407, 4154-4159.

48. M. Hong, Z.-G. Chen, L. Yang, T. C. Chasapis, S. D. Kang, Y. Zou, G. J. Auchterlonie, M. G. Kanatzidis, G. J. Snyder and J. Zou, Journal of Materials Chemistry A, 2017, 5, 10713-10721.

49. D. J. Bergman and O. Levy, Journal of Applied Physics, 1991, 70, 6821-6833.

50. D. J. Bergman and L. G. Fel, Journal of Applied physics, 1999, 85, 8205-8216.

51. M. H. Lee, J. H. Yun, G. Kim, J. E. Lee, S.-D. Park, H. Reith, G. Schierning, K. Nielsch, W. Ko and A.-P. Li, ACS nano, 2019, 13, 3806-3815.

52. P. Jost, H. Volker, A. Poitz, C. Poltorak, P. Zalden, T. Schäfer, F. R. Lange, R. M. Schmidt, B. Holländer and M. R. Wirtssohn, Advanced functional materials, 2015, 25, 6399-6406.

53. L. Zhao, S. M. K. N. Islam, J. Wang, D. L. Cortie, X. Wang, Z. Cheng, J. Wang, N. Ye, S. Dou and X. Shi, Nano Energy, 2017, 41, 164-171.

54. R. Nunna, P. Qiu, M. Yin, H. Chen, R. Hanus, Q. Song, T. Zhang, M.-Y. Chou, M. T. Agne and J. He, Energy \& Environmental Science, 2017, 10, 1928-1935.

55. M. Li, D. L. Cortie, J. Liu, D. Yu, S. M. K. N. Islam, L. Zhao, D. R. Mitchell, R. A. Mole, M. B. Cortie and S. Dou, Nano energy, 2018, 53, 993-1002.

56. M. Li, S. M. K. N. Islam, S. Dou and X. Wang, Journal of Alloys and Compounds, 2018, 769, 59-64.

57. P. Qiu, M. T. Agne, Y. Liu, Y. Zhu, H. Chen, T. Mao, J. Yang, W. Zhang, S. M. Haile and W. G. Zeier, Nature communications, 2018, 9, 2910.

58. Y. Zhang, X. Wang, W. Yeoh, R. Zheng and C. Zhang, Applied Physics Letters, 2013, 102, 031909.

59. H. Tang, F.-H. Sun, J.-F. Dong, H.-L. Zhuang, Y. Pan and J.-F. Li, Nano energy, 2018, 49, $267-$ 273. 
60. S. Sassi, C. Candolfi, J.-B. Vaney, V. Ohorodniichuk, P. Masschelein, A. Dauscher and B. Lenoir, Applied Physics Letters, 2014, 104, 212105.

61. G. J. Snyder and E. S. Toberer, Nature Materials, 2008, 7, 105-114.

62. D. Bansal, J. Hong, C. W. Li, A. F. May, W. Porter, M. Y. Hu, D. L. Abernathy and O. Delaire, Physical Review B, 2016, 94, 054307.

63. C. Cavallari, D. Pontiroli, M. Jiménez-Ruiz, A. Ivanov, M. Mazzani, M. Gaboardi, M. Aramini, M. Brunelli, M. Riccò and S. Rols, Journal of Physics: Conference Series, 2014, 554, 012009.

64. S. Johnsen, J. He, J. Androulakis, V. P. Dravid, I. Todorov, D. Y. Chung and M. G. Kanatzidis, Journal of the American Chemical Society, 2011, 133, 3460-3470.

65. M. Cutler, J. Leavy and R. Fitzpatrick, Physical Review, 1964, 133, A1143.

66. H. Sun, X. Jia, P. Lv, L. Deng, X. Guo, Y. Zhang, B. Sun, B. Liu and H. Ma, RSC Advances, 2015, 5, 61324-61329. 